\title{
Análisis de residuos de herbicidas en aguas procedentes de Navarra mediante LC-MS/MS
}

\section{LC-MS/MS analysis of herbicide residues in waters from Navarre}

\author{
P. Fraile ${ }^{1}$, M. Izu ${ }^{2}$, I. Sáiz ${ }^{2}$, J. Castiella ${ }^{1}$, J.A. Pérez de Ciriza ${ }^{2}$
}

\section{RESUMEN}

Fundamento. El objetivo del trabajo fue obtener información acerca de los residuos de herbicidas (imazametabenz, simazina, isoproturon, clortoluron, metribuzina, atrazina, cianazina, terbutrina, propanil, terbutilazina, alacloro y pendimetalina) presentes en aguas procedentes de Navarra.

Material y métodos. Se tomó un total de 465 muestras de agua; de ellas, 378 se obtuvieron en 141 abastecimientos; las 87 restantes procedieron de 37 fuentes, manantiales y sondeos situados en zonas agrícolas del sur de Navarra. La técnica empleada fue LC-MS/MS.

Resultados. Se detectaron residuos de herbicidas en el $33 \%$ de los abastecimientos, y su concentración superó el límite normativo en el $8 \%$ de ellos. Se encontraron residuos de herbicidas en el $86 \%$ de las muestras de pozos y fuentes de zonas agrícolas del sur de Navarra. El número de abastecimientos con residuos de herbicidas aumentó de norte a sur de Navarra. No obstante, el porcentaje de abastecimientos en los que la concentración de herbicidas superó los límites fue mayor en la zona centro de Navarra que en la zona sur y norte. Imazametabenz fue el herbicida más detectado en las muestras de las zonas norte, centro y sur. Atrazina, terbutilazina e imazametabenz se detectaron preferentemente en las muestras de la zona sur.

Conclusiones. Las diferencias entre las tres zonas de Navarra pueden explicarse atendiendo a las diferentes prácticas agrícolas de cada región. No se detectaron concentraciones de herbicidas superiores a los límites normativos en ninguna de las muestras de agua procedentes de las poblaciones mayoritarias de Navarra.

Palabras clave. Herbicidas. Residuos. Aguas. Navarra. LC-MS/MS.

\begin{abstract}
Background. The aim of the study was to obtain information on the residues of herbicides (imazametabenz, simazine, isoproturon, clortoluron, metribuzine, atrazine, cianazine, terbutrin, propanil, terbutilazine, alachlor and pendimetalin) present in water proceeding from Navarre.
\end{abstract}

Material and methods. A total of 465 samples of water were taken: 378 were taken from 141 water supplies; the remaining 87 proceeded from fountains, springs and borings in agricultural areas in the south of Navarre. The technique employed was LC-MS/MS.

Results. Herbicide residues were detected in $33 \%$ of the water supplies, and their concentration exceeded the normative limit in $8 \%$ of them. Herbicide residues were found in $86 \%$ of the samples from wells and fountains of the agricultural areas of the south of Navarre. The number of water supplies with herbicide residues increased from north to south. However, the percentage of water supplies in which the concentration of herbicides exceeded the limits was greater in the centre of Navarre than in the north and south. Imazametabenz was the herbicide most detected in the samples from the northern, central and southern areas. Atrazine, terbutilazine and imazametabenz were most detected in the samples of the southern area.

Conclusions. The differences amongst the three areas of Navarre can be explained as being due to the different agricultural practices of each area. Concentrations of herbicides exceeding the normative limits were not detected in any of the water samples proceeding from the larger towns of Navarre.

Key words. Herbicides. Residues. Water. Navarre. LCMS/MS.
1. Departamento de Desarrollo Rural y Medio Ambiente. Gobierno de Navarra.

2. Instituto de Salud Pública. Departamento de Salud. Gobierno de Navarra.

Este trabajo se ha realizado dentro del proyecto de investigación "Aplicación de la cromatografía de líquidos acoplada a espectrometría de masas con triple cuadrupolo al control de la seguridad alimentaria. Creación de una base de datos espectral de compuestos orgánicos de interés sanitario", concedido mediante resolución 1598/2005 del Departamento de Salud del Gobierno de Navarra.

\author{
Correspondencia \\ Pablo Fraile Jiménez de Maquirriain \\ Laboratorio de Calidad Agroalimentaria \\ Departamento de Desarrollo Rural y Medio \\ Ambiente \\ Avda. Serapio Huici s/n \\ 31610 Villava (Navarra) \\ Tfno. 848424863 \\ E-mail: pfrailej@cfnavarra.es \\ Recepción: 23 de marzo de 2009 \\ Aceptación provisional: 11 de junio de 2009 \\ Aceptación definitiva: 23 de julio de 2009
}




\section{INTRODUCCIÓN}

Los herbicidas procedentes de los tratamientos fitosanitarios pueden contaminar el suelo, como consecuencia de su forma de aplicación. Las características físico-químicas de las materias activas de las formulaciones de herbicidas, las propiedades de los suelos a los que se aplican y otros factores como el régimen de lluvias, la topología y los vientos dominantes de la zona, condicionan la posible migración de las mismas al medio acuático $^{1}$. En los Estados Unidos, se han detectado plaguicidas en aguas procedentes de una amplia variedad de entornos hidrogeológicos y de cultivos, incluyendo los acuíferos utilizados para el suministro de agua para consumo humano ${ }^{2}$. En los países europeos también se han detectado plaguicidas en las aguas. Así, diferentes autores han puesto de manifiesto la contaminación de las aguas en el Reino Uni$\mathrm{do}^{3}$, Alemania $^{4}$, Italia $^{5}$, España $^{6}$, Francia $^{7}$ y Holanda ${ }^{8}$. La normativa española para aguas de consumo establece un límite máximo de $0,1 \mu \mathrm{g} / \mathrm{L}$ para cada plaguicida individual y de $0,5 \mu \mathrm{g} / \mathrm{L}$ para la suma de las concentraciones de plaguicidas. Navarra es una comunidad autónoma que, en poco más de $10.400 \mathrm{~km}^{2}$, presenta una gran diversidad de climas, que varían desde el árido hasta el muy húmedo. Consecuentemente, existe una amplia variación en los cultivos y en las prácticas agrícolas asociadas, lo que añade interés al estudio de los residuos generados, y su relación con las diferentes zonas climáticas.

De acuerdo con los datos del Instituto Técnico de Gestión Agrícola de Navarra, se aplican al año en la Comunidad Foral aproximadamente $180.000 \mathrm{~kg}$ de herbicidas. En un estudio previo realizado en Navarra en el año $2002^{9}$, se analizaron residuos de 7 herbicidas en muestras de agua procedentes de 101 localidades. Sólo se detectó un herbicida (cianazina) en una de las muestras de agua analizadas; la concentración hallada fue de $0,4 \pm$ $0,2 \mu \mathrm{g} / \mathrm{L}$. Debe considerarse que en el año 2003 , con posterioridad al inicio del trabajo citado, se recogió en la legislación un límite máximo de $0,1 \mu \mathrm{g} / \mathrm{L}$ (aunque estaba ya establecido) para cada plaguicida individual en aguas de consumo. Los límites de cuantificación obtenidos mediante HPLC-UV en el estudio citado para algunos de los compuestos eran excesivamente altos. Quedó patente, por tanto, la conveniencia de realizar investigaciones adicionales que permitieran detectar grupos más amplios de compuestos por debajo de los límites normativos vigentes. Autores como Hernández y col ${ }^{10}$, Sancho y $\operatorname{col}^{11}$, Kuster y $\operatorname{col}^{12}$, han propuesto la utilización de nuevas técnicas analíticas como LC-MS/MS para el análisis de residuos de herbicidas. La selectividad y sensibilidad de esta técnica son excelentes, lo que la hacen muy adecuada para la detección de residuos a concentraciones muy bajas.

Los objetivos de este trabajo han sido:

- Aplicar la técnica LC-MS/MS para la detección de residuos de herbicidas en agua a niveles inferiores a 0,1 $\mu \mathrm{g} / \mathrm{L}$.

- Desarrollar un método para compuestos de amplia utilización en Navarra. Los productos seleccionados fueron: imazametabenz, simazina, isoproturon, clortoluron, metribuzina, atrazina, cianazina, terbutrina, propanil, terbutilazina, alacloro, pendimetalina y glifosato.

- Conocer los residuos de herbicidas presentes en las aguas procedentes de las diferentes zonas climáticas de Navarra y estudiar sus eventuales variaciones espaciales y temporales.

\section{MATERIAL Y MÉTODOS}

\section{Muestras}

Las muestras de agua se han clasificado atendiendo a su origen geográfico. Con este fin, se han diferenciado tres zonas de Navarra: norte, centro y sur. Se han incluido dentro de la zona norte de Navarra las localidades situadas al norte de la ciudad de Pamplona. Las localidades cuya latitud está comprendida entre las de Pamplona 
y de la ciudad de Tafalla se han encuadrado en la zona centro. Las cuencas prepirenaicas de Pamplona y de Aoiz-Lumbier se consideran usualmente dentro de la Montaña de Navarra; no obstante, por sus características agrícolas, en este estudio, estas cuencas se han encuadrado en la zona centro. Las poblaciones situadas al sur de Tafalla se han considerado pertenecientes a zona sur. En los dos años del estudio (2006 y 2007) se tomó un total de 465 muestras de agua. De ellas, 378 se obtuvieron en 141 abastecimientos de agua de consumo humano (tratadas con cloro), alimentados por aguas superficiales y subterráneas. Las 87 muestras restantes (no cloradas) procedieron de 37 fuentes, manantiales y sondeos situados en zonas agrícolas del sur de Navarra; estas muestras se tomaron durante el año 2006 y formaban parte de un estudio más amplio de carácter hidrogeológico. En buena parte de las localizaciones, se repitieron los muestreos en diferentes meses con el fin de conocer la variación temporal de los residuos de herbicidas. La distribución de las muestras por zonas se realizó atendiendo a las prácticas agrícolas de cada una de ellas. De este modo, en la zona norte se tomaron un total de 52 muestras, frente a 211 en la zona centro y 202 en la zona sur.

\section{Patrones cromatográficos y reactivos}

Se utilizaron patrones de imazametabenz, simazina, isoproturon, clortoluron, metribuzina, atrazina, cianazina, terbutrina, propanil, terbutilazina, alacloro, pendimetalina y glifosato, todos ellos suministrados por la firma Dr. Ehrenstorfer (Ausburgo, Alemania). Estos patrones se manipularon siguiendo las indicaciones de seguridad del fabricante, utilizando medios de protección personal y campanas de seguridad. Se utilizó agua calidad HPLC obtenida por ósmosis inversa en un sistema de purificación Millipore (Billerica, MA, USA). También se empleó metanol, acetonitrilo calidad HPLC y ácido fórmico
(89-91\%) para análisis de la firma Merck (Darmstadt, Alemania).

\section{Equipos}

Los análisis se realizaron en un cromatógrafo de líquidos (LC) acoplado a un detector de espectrometría de masas en tandem (MS/MS), modelo Micromass Quantum Quattro. Este equipo consistió en un sistema de bombeo y de inyección automática 2795 Alliance HT (Waters, Milford, Massachussets, USA), y un detector Waters Micromass Quattro Micro API (Allrincham Chesire, UK), equipado con Z-spray. Se seleccionó electrospray (ESI) como modo de ionización. Se utilizó una columna de fase reversa Waters Atlantis dC 18 de 2,1 x $100 \mathrm{~mm}$ y un tamaño de partícula de $3 \mu \mathrm{m}$. La columna se termostatizó a $30^{\circ} \mathrm{C}$.

\section{Método de purificación, concentración y análisis cromatográfico}

Se activó un cartucho Oasis HLB de 60 mg (Waters, Barcelona, España) pasando sucesivamente $3 \mathrm{~mL}$ de metanol y $3 \mathrm{~mL}$ de agua. Se pasaron a continuación $20 \mathrm{~mL}$ de la muestra de agua a un flujo máximo de 4 $\mathrm{ml} / \mathrm{min}$. Seguidamente, se lavó con $1 \mathrm{~mL}$ de agua ultrapura. Se colocó un tubo colector, y se eluyó con 1,2 mL de metanol. Se añadieron $0,8 \mathrm{~mL}$ de agua al $0,1 \%$ de ácido fórmico al tubo. El tubo se agitó en un agitador vortex, y el líquido se filtró a través de un filtro de $0,22 \mu \mathrm{m}$ (Teknokroma, Barcelona, España). El filtrado se encapsuló en viales de inyector automático; por último, se inyectaron $20 \mu \mathrm{L}$ del extracto en el equipo LC-MS-MS. La composición de la fase móvil A fue una mezcla de acetonitrilo/ácido fórmico $(99,9: 0,1 \mathrm{v} / \mathrm{v})$; la fase $B$ fue una mezcla de agua/ácido fórmico $(99,9: 0,1 \mathrm{v} / \mathrm{v})$. El caudal fue $0,25 \mathrm{~mL} / \mathrm{min}$. Se utilizó el siguiente gradiente: $0 \mathrm{~min}$, $25 \%$ de A; 6 min, $80 \%$ de A; 10 min, 25\% de A. La separación cromatográfica de los herbicidas se completó en 12 min.

En cuanto a las condiciones de operación del detector, la temperatura de la 
fuente de iones se fijó en $100{ }^{\circ} \mathrm{C}$ y el voltaje del capilar en $3,00 \mathrm{kV}$. El gas de colisión fue argón, a una presión de aproximadamente $3,5 \times 10^{-3}$ mbar en la célula de colisión. El caudal del gas de ionización (nitrógeno) a presión atmosférica fue $650 \mathrm{~L} \mathrm{~h}^{-1}$, y la temperatura de desolvatación $350^{\circ} \mathrm{C}$. El modo de electrospray fue positivo para todos los compuestos analizados. Para analizar los herbicidas mediante MS/MS, el voltaje de cono se fijó al valor optimizado para cada compuesto.

La tabla 1 resume los datos cromatográficos y espectrales obtenidos tras el desarrollo y optimización de los parámetros instrumentales. La confirmación espectral de los picos obtenidos a los tiempos de retención de los herbicidas consistió en la verificación de las transiciones específicas, seguida de la comprobación de las abundancias relativas de cada ión. Para la cuantificación se utilizaron rectas de calibración obtenidas con patrones de herbicidas de concentraciones comprendidas entre los límites de cuantificación respectivos y $0,50 \mu \mathrm{g} / \mathrm{L}$. El método de análisis se validó mediante un procedimiento interno. Inicialmente, se estudió la linealidad del detector para cada uno de los compuestos, inyectando en el sistema LC-MS/ MS disoluciones de herbicidas a concen-

Tabla 1. Parámetros cromatográficos y espectrales fijados para el análisis de herbicidas.

\begin{tabular}{|c|c|c|c|c|}
\hline Compuesto & $\begin{array}{c}\text { Tiempo retención } \\
\text { (min) }\end{array}$ & $\begin{array}{c}\text { Voltaje cono } \\
\text { (V) }\end{array}$ & $\begin{array}{c}\text { Energía colisión } \\
\text { (eV) }\end{array}$ & Transición \\
\hline \multirow{2}{*}{ Imazametabenz } & \multirow{2}{*}{4,59} & 30 & 20 & $289-86$ \\
\hline & & 30 & 30 & $289-229$ \\
\hline \multirow{2}{*}{ Simazina } & \multirow{2}{*}{5,74} & 30 & 20 & $202-124$ \\
\hline & & 30 & 20 & 202-132 \\
\hline \multirow{2}{*}{ Cianazina } & \multirow{2}{*}{5,95} & 30 & 15 & $241-214$ \\
\hline & & 30 & 20 & $241-174$ \\
\hline \multirow{2}{*}{ Metribuzina } & \multirow{2}{*}{6,09} & 30 & 20 & $215-187$ \\
\hline & & 30 & 20 & $215-171$ \\
\hline \multirow{2}{*}{ Terbutrina } & \multirow{2}{*}{6,21} & 30 & 35 & $242-71$ \\
\hline & & 30 & 20 & $242-186$ \\
\hline \multirow{2}{*}{ Clortoluron } & \multirow{2}{*}{6,58} & 25 & 15 & $213-72$ \\
\hline & & 25 & 25 & 213-140 \\
\hline \multirow{2}{*}{ Atrazina } & \multirow{2}{*}{6,75} & 30 & 15 & $216-174$ \\
\hline & & 30 & 20 & 216-132 \\
\hline \multirow{2}{*}{ Isoproturon } & \multirow{2}{*}{6,84} & 40 & 15 & $207-72$ \\
\hline & & 46 & 19 & 207-165 \\
\hline \multirow{2}{*}{ Propanil } & \multirow{2}{*}{7,65} & 35 & 15 & $218-162$ \\
\hline & & 35 & 25 & 218-127 \\
\hline \multirow{2}{*}{ Terbutilazina } & \multirow{2}{*}{7,83} & 20 & 15 & $230-174$ \\
\hline & & 20 & 25 & $230-132$ \\
\hline \multirow{2}{*}{ Alacloro } & \multirow{2}{*}{8,80} & 15 & 10 & $270-238$ \\
\hline & & 15 & 25 & $270-162$ \\
\hline \multirow{2}{*}{ Pendimetalina } & \multirow{2}{*}{11,31} & 25 & 20 & $282-212$ \\
\hline & & 25 & 25 & $282-194$ \\
\hline
\end{tabular}


traciones crecientes. Se construyeron 10 rectas para cada compuesto con 5 niveles de concentración, y la linealidad se evaluó mediante el coeficiente de regresión de la recta promedio. El límite de detección (LD) se calculó inyectando 10 muestras blanco para cada compuesto; el LD se calculó como 5 veces la desviación estándar de las medidas. El límite de cuantificación (LOQ) se calculó como 2 veces el LD. Para evaluar la exactitud del método, se utilizó el porcentaje de recuperación. Para calcularlo, se prepararon 10 muestras de agua adicionadas con concentraciones de herbicidas correspondientes a los puntos extremos (LOQ y $0,5 \mu \mathrm{g} / \mathrm{L}$ ) del rango lineal de cada compuesto. La precisión del método se calculó mediante los coeficientes de variación (CV) obtenidos a partir de las muestras adicionadas. Los resultados obtenidos para los parámetros citados se han resumido en la tabla 2. En la figura 1 se muestra un cromatograma obtenido en modo MRM, correspondiente a una muestra de agua adicionada con herbicidas a una concentración de $0,50 \mu \mathrm{g} / \mathrm{L}$.

Tabla 2. Parámetros obtenidos en la validación del método de análisis.

\begin{tabular}{|c|c|c|c|c|c|}
\hline Compuesto & $\begin{array}{c}\text { Intervalo } \\
\text { linealidad } \\
(\mu \mathrm{g} / \mathrm{L})\end{array}$ & $\begin{array}{l}\text { Coeficiente } \\
\text { de regresión } \\
\text { (r) }\end{array}$ & $\begin{array}{c}\text { LOQ } \\
(\mu \mathrm{g} / \mathrm{L})\end{array}$ & $\begin{array}{c}\text { Precisión (CV) } \\
\text { (nivel LOQ- } \\
\text { nivel } 0,5 \mu \mathrm{g} / \mathrm{L} \text { ) }\end{array}$ & $\begin{array}{c}\text { Recuperación } \\
\text { (nivel LOQ- } \\
\text { nivel } 0,5 \mu \mathrm{g} / \mathrm{L} \text { ) }\end{array}$ \\
\hline Terbutrina & $0,01-0,50$ & 0,9997 & 0,01 & $21-0,3 \%$ & $80-87 \%$ \\
\hline Cianazina & $0,03-0,50$ & 0,9998 & 0,03 & $31-0,3 \%$ & $87-95 \%$ \\
\hline Atrazina & $0,01-0,50$ & 0,9998 & 0,01 & $28-0,3 \%$ & $81-94 \%$ \\
\hline Metribuzina & $0,01-0,50$ & 0,9999 & 0,01 & $41-4,4 \%$ & $105-90 \%$ \\
\hline Simazina & $0,02-0,50$ & 0,9998 & 0,02 & $42-0,3 \%$ & $79-92 \%$ \\
\hline Pendimetalina & $0,09-0,50$ & 0,9984 & 0,09 & $25-0,4 \%$ & $27-44 \%$ \\
\hline Alacloro & $0,01-0,50$ & 0,9999 & 0,01 & $49-0,4 \%$ & $54-88 \%$ \\
\hline Terbutilazina & $0,01-0,50$ & 0,9997 & 0,01 & $14-0,2 \%$ & $84-91 \%$ \\
\hline Propanil & $0,05-0,50$ & 0,9997 & 0,05 & $38-0,4 \%$ & $78-100 \%$ \\
\hline Clortoluron & $0,05-0,50$ & 0,9990 & 0,05 & $9-0,3 \%$ & $102-100 \%$ \\
\hline Isoproturon & $0,05-0,50$ & 0,9998 & 0,05 & $11-1,2 \%$ & $83-100 \%$ \\
\hline Imazametabenz & $0,01-0,50$ & 0,9998 & 0,01 & $15-0,2 \%$ & $80-96 \%$ \\
\hline
\end{tabular}




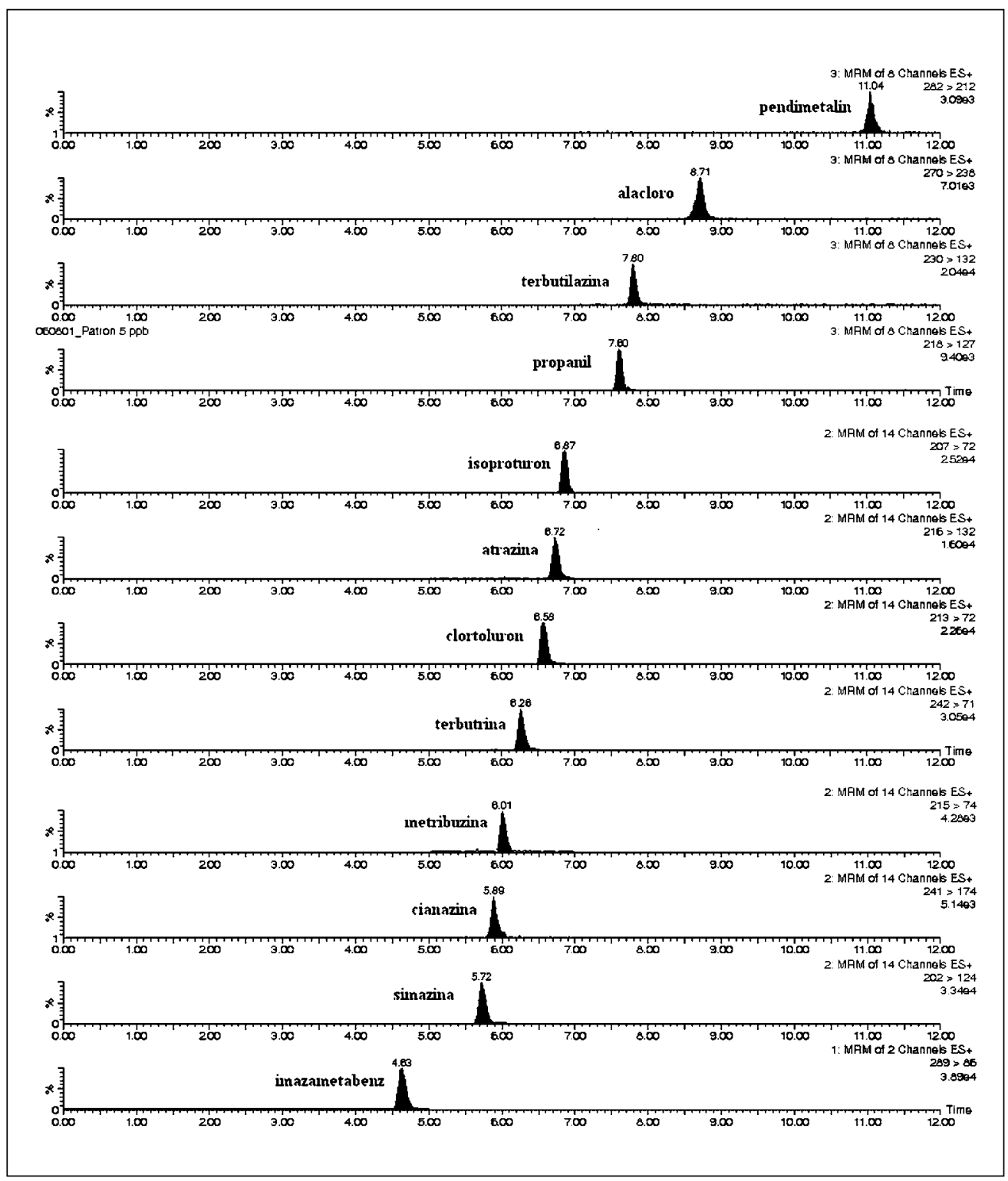

Figura 1. Cromatograma obtenido en modo MRM, correspondiente a una muestra de agua adicionada con herbicidas a una concentración de $0,50 \mu \mathrm{g} / \mathrm{L}$.

\section{RESULTADOS}

El método descrito pudo aplicarse a la detección de los residuos de 12 herbicidas. Entre los objetivos del trabajo se había incluido la detección de glifosato, pero no se consiguió analizar este compuesto junto con el resto de herbicidas del método multirresiduo. Los límites de cuantificación obtenidos para 11 de los 12 compuestos estuvieron en el intervalo 0,01-0,05 $\mu \mathrm{g} / \mathrm{L}$. El límite de cuantificación de pendimetalina fue $0,09 \mu \mathrm{g} / \mathrm{L}$. 


\section{Residuos en aguas de consumo humano}

En la tabla 3 se presentan los datos sobre los residuos de herbicidas en 141 abastecimientos de agua de Navarra. Puede observarse que el porcentaje de abastecimientos en los que se detectó la presencia de herbicidas aumentó de norte a sur. No obstante, el porcentaje de abastecimientos no conformes, es decir, aquellos en los que la concentración de herbicidas superó los límites establecidos (aunque sólo lo hiciera en uno de los muestreos estacionales), fue mayor en la zona centro que en la zona sur y en la norte. La zona norte destacó por la escasa presencia de residuos de herbicidas en sus abastecimientos. No se detectaron concentraciones de herbicidas supe- riores a los límites normativos en ninguna de las muestras de agua procedentes de las poblaciones mayoritarias de Navarra, con gestión mancomunada del suministro de agua. En la mayor parte de los abastecimientos, se tomaron varias muestras en el transcurso del estudio. Las concentraciones totales de herbicidas halladas en las muestras se presentan en la tabla 4 . El porcentaje de muestras positivas en las zonas centro y sur fue más de tres veces superior al de la zona norte. En las zonas centro y sur, los porcentajes de muestras en las que se detectó residuos de herbicidas fueron similares. No obstante, se observan diferencias notables en las concentraciones medias de las muestras positivas; puede establecerse para estas concentraciones la pauta general: centro $>$ norte $>$ sur.

Tabla 3. Residuos de herbicidas en abastecimientos de agua de Navarra.

\begin{tabular}{lccc}
\hline Origen & № abastecimientos & $\begin{array}{c}\text { Abastecimientos } \\
\text { con residuos }\end{array}$ & $\begin{array}{c}\text { Abastecimientos } \\
\text { no conformes }\end{array}$ \\
\hline Norte & 42 & $7 \%$ & $5 \%$ \\
Centro & 56 & $41 \%$ & $11 \%$ \\
Sur & 43 & $46 \%$ & $7 \%$ \\
Total Navarra & 141 & $33 \%$ & $8 \%$ \\
\hline
\end{tabular}

Tabla 4. Concentración total de herbicidas en muestras de agua de consumo humano.

\begin{tabular}{lccc}
\hline \multicolumn{1}{c}{ Origen } & № muestras & \% positivas & $\begin{array}{c}\text { Concentración media } \\
\text { positivos } \\
(\mu \mathbf{g} / \mathbf{L} \pm \mathbf{s})\end{array}$ \\
\hline Norte & 52 & $11 \%$ & $0,21 \pm 0,03$ \\
Centro & 212 & $37 \%$ & $0,76 \pm 0,07$ \\
Sur & 114 & $34 \%$ & $0,04 \pm 0,01$ \\
Total Navarra & 378 & $32 \%$ & $0,17 \pm 0,02$ \\
\hline
\end{tabular}

En lo que se refiere a los herbicidas detectados en cada zona de Navarra, la figura 2 muestra los porcentajes de detección de los herbicidas presentes en las muestras positivas de cada demarcación. Se observa que imazametabenz fue el herbicida cuyos residuos se detectaron con mayor frecuencia en las muestras de las zonas norte, centro y sur. En la zona sur, le siguieron en orden de frecuencia tres compuestos pertenecientes al grupo de las triazinas: terbutilazina, atrazina y simazina. En la zona centro, se detectaron residuos de terbutilazina y simazina, pero no de atrazina. De los compuestos pertenecientes a la familia de las ureas sustituidas, isoproturon apareció en muestras de las zonas norte y centro y clortoluron 
lo hizo en aguas de la zona centro. Sólo se detectó propanil en una muestra de la zona centro. No se detectaron residuos de cianazina, terbutrina, metribuzina, alacloro y pendimetalina en ninguna de las muestras del estudio.

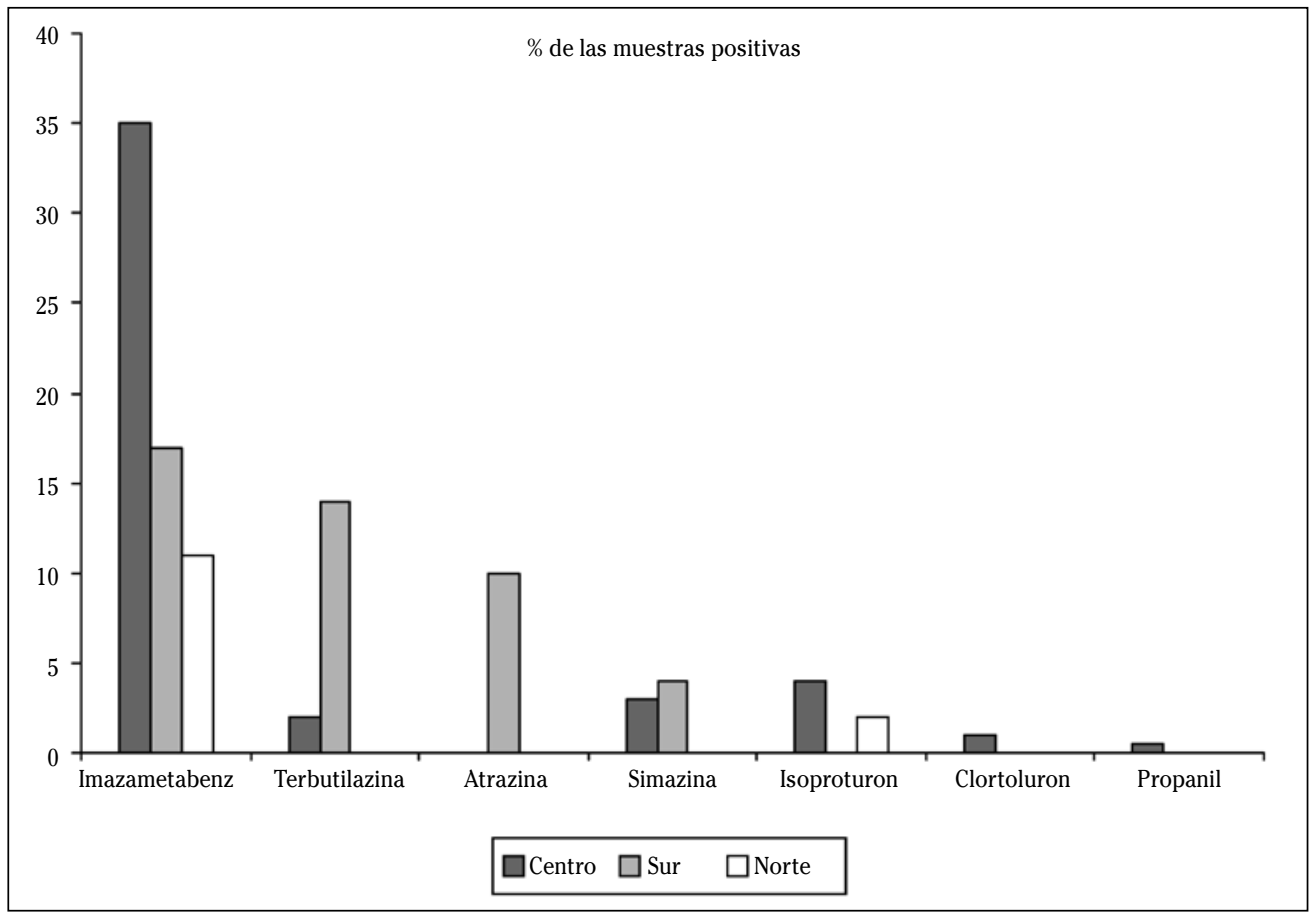

Figura 2. Herbicidas detectados en las muestras positivas de abastecimientos de agua.

Hasta ahora, la presentación de los resultados se ha referido a la suma de las concentraciones de los herbicidas. Esta información puede desglosarse en las concentraciones individuales de los compuestos incluidos en el estudio. Para ello, la tabla 5 presenta las concentraciones medias de los herbicidas detectados en las muestras positivas. De los datos de esta tabla se desprende que, de forma general, en la zona centro se detectó una mayor variedad de compuestos que en las zonas sur y norte. Imazametabenz fue el compuesto más detectado; en un abastecimiento de la zona centro se encontró a una concentración de $7,03 \pm 0,09 \mu \mathrm{g} / \mathrm{L}$, que es la más alta alcanzada en las tres zonas. En las zonas norte y centro, el compuesto detectado a una mayor concentración media fue isoproturon, y todas las muestras positivas para este compuesto superaron los límites legales. Atrazina, terbutilazina e imazametabenz se detectaron en un mayor número de muestras de la zona sur, y sus concentraciones medias fueron bajas. Aunque las concentraciones medias de terbutilazina y atrazina halladas en la zona sur no superaron los $0,1 \mu \mathrm{g} / \mathrm{L}$ reglamentados, sí que hubo muestras que superaron ligeramente este límite. Propanil tan sólo se detectó en una muestra de la zona centro, a baja concentración.

Para valorar la evolución temporal de la concentración total de residuos, se han seleccionado las localidades de las zonas norte, centro y sur en las que se detectaron las mayores concentraciones de herbicidas. En estos abastecimientos se realizaron muestreos sucesivos, cuyos resultados analíticos 
se presentan en las figuras 3 y 4 . Los abastecimientos de las zonas norte y centro captan sus aguas de manantiales, mientras que el de la zona sur toma sus aguas mediante bombeo de un pozo. En el punto de muestreo de la zona norte con mayor concentración de residuos (Fig. 3a), la concentración más alta se alcanzó en invierno; la concentración de los herbicidas detectados (clortoluron e imazametabenz) disminuyó durante los meses de marzo y abril, pero la suma de las concentraciones detectadas superaron todavía los límites legales. En el mes de mayo, los niveles detectados fueron inferiores a los normativos y esta situación se mantuvo hasta el mes de octubre en los sucesivos muestreos. En el abastecimiento de la zona centro seleccionado (Fig. 3b) se encontraron residuos de imazametabenz. La evolución fue similar a la expuesta para la zona norte: se

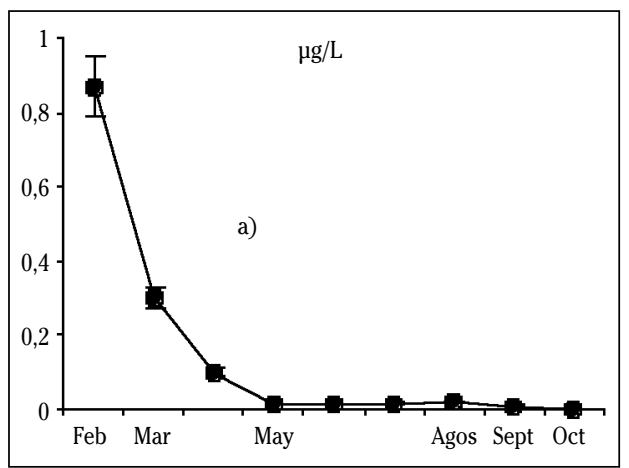

detectó la máxima concentración en invierno, y ya avanzada la primavera (mayo), los niveles de residuos fueron bajos. En la localidad seleccionada de la zona sur, se detectó atrazina, terbutilazina, simazina e imazametabenz. La concentración total de herbicidas fue menor que en los abastecimientos de las zonas norte y centro; además, su evolución fue diferente a las de las zonas anteriores, tal como se muestra en la figura 4 . En este abastecimiento, la mayor concentración de residuos se encontró en el mes de febrero; no obstante, no se produjo una disminución notable de la concentración de herbicidas en las muestras sucesivas. Entre los meses de marzo a mayo, la concentración de residuos fue prácticamente la misma. A partir de mayo, se produjo una reducción y los niveles se mantuvieron por debajo de los límites normativos.

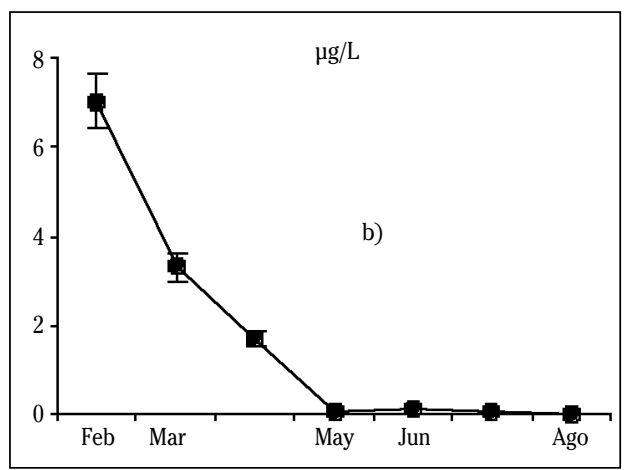

Figura 3. Evolución (año 2007) de la concentración total de residuos de herbicidas; a) en un abastecimiento de la zona norte, b) en un abastecimiento de la zona centro. Se hace constar los meses en los que se tomaron las muestras.

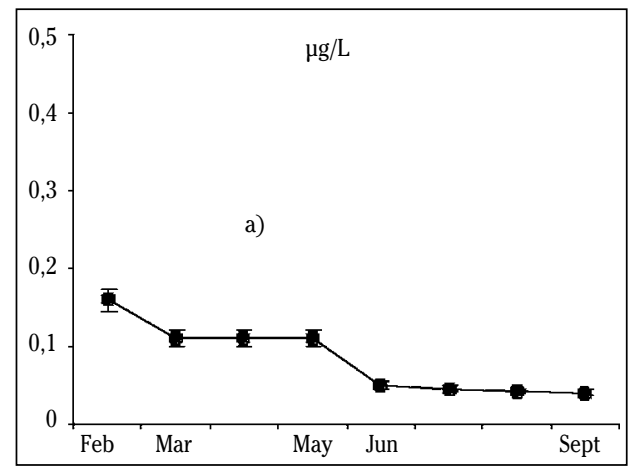

Figura 4. Evolución (año 2006) de la concentración total de residuos de herbicidas en un abastecimiento de la zona sur. Se hace constar los meses en los que se tomaron las muestras. 


\section{Residuos en fuentes y pozos de zonas agrícolas}

En el estudio se incluyeron muestras de agua que no procedían de abastecimientos de agua de consumo y que fueron tomadas en 37 fuentes, pozos y sondeos situados en zonas agrícolas del sur de Navarra. En ellas se constató un elevado porcentaje (86\%) de muestras con residuos de herbicidas. La concentración media hallada en las muestras positivas de estas zonas agrícolas $(0,14 \pm 0,2 \mu \mathrm{g} / \mathrm{L})$ fue superior a la de los abastecimientos positivos de la zona sur $(0,04 \pm 0,01 \mu \mathrm{g} / \mathrm{L})$. Las concentraciones de las muestras positivas estuvieron dentro del intervalo $0,01-1,42 \mu \mathrm{g} / \mathrm{L}$. Los herbicidas detectados en las muestras positivas se recogen en la figura 5. En este tipo de muestras, se observó una mayor variedad de compuestos que en las muestras procedentes de abastecimientos de la zona sur (Fig. 2).

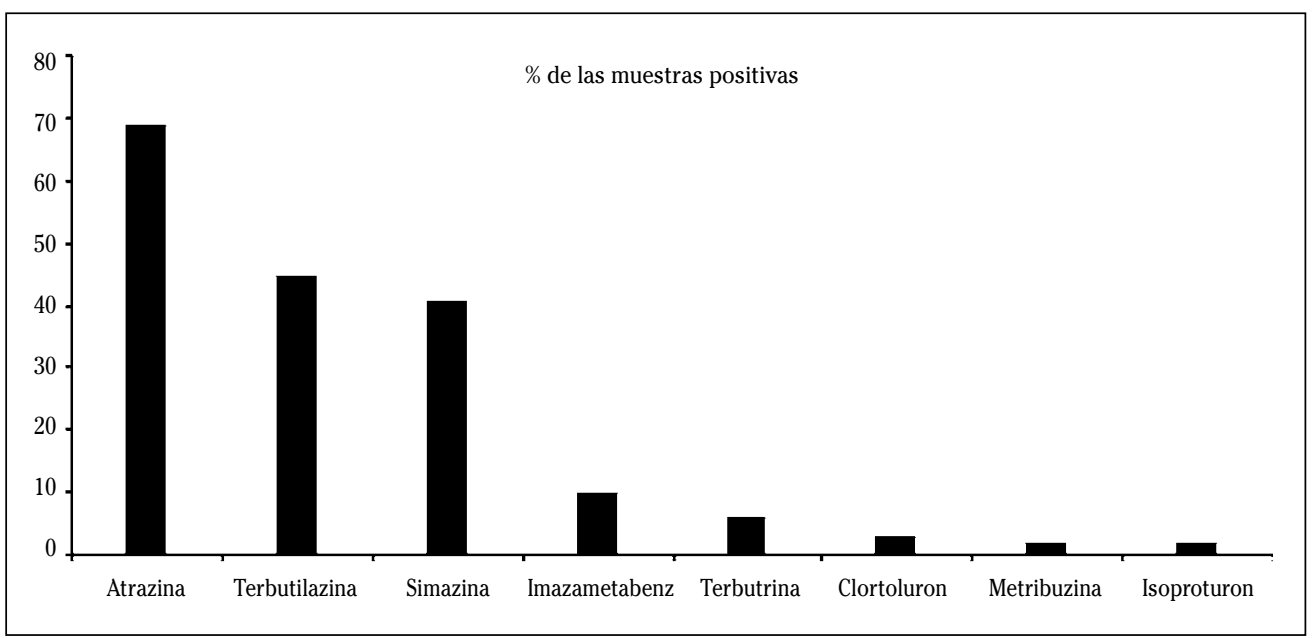

Figura 5. Herbicidas detectados en las muestras de pozos y fuentes situados en zonas agrícolas del sur de Navarra.

Los compuestos más detectados pertenecen al grupo de las triazinas; el herbicida que se detectó en la mayor parte de las muestras positivas fue atrazina, seguido de terbutilazina y simazina. Se presentan a continuación, en la tabla 6 , las concentraciones de herbicidas halladas en las muestras de zonas agrícolas con residuos de herbicidas. Los datos indican que los valores medios de concentración fueron inferiores al límite de $0,1 \mu \mathrm{g} / \mathrm{L}$ (a excepción de de clortoluron). No obstante, todas estas concentraciones medias son superiores a las encontradas en los abastecimientos de la zona sur (Tabla 5). También, en comparación con los resultados de los abastecimientos, son mayores los extremos superiores de los rangos de concentración de terbutilazina, atrazina, y simazina. Debe mencionarse que, en una muestra de un pozo de la zona sur se detectaron residuos de 7 compuestos (simazina, terbutilazina, terbutrina, metribuzina, clortoluron, isoproturon, imazametabenz), cuya suma de concentraciones llegó a alcanzar 0,72 \pm 0,06 $\mu \mathrm{g} / \mathrm{L}$ (Fig. 6). 
Tabla 5. Concentración media $(\mu \mathrm{g} / \mathrm{L} \pm \mathrm{s})$ de herbicidas en muestras positivas procedentes de abastecimientos. Entre paréntesis figura el intervalo de concentraciones de las muestras.

\begin{tabular}{cccccccc}
\hline & Imazametabenz & Terbutilazina & Atrazina & Simazina & Isoproturon & Clortoluron & Propanil \\
\hline $\mathrm{C}$ & $0,65 \pm 0,03$ & $0,02 \pm 0,01$ & - & $0,13 \pm 0,02$ & $1,09 \pm 0,03$ & $0,15 \pm 0,01$ & $0,08 \pm 0,01$ \\
$\mathrm{E}$ & $(0,01-7,03)$ & $(0,01-0,03)$ & - & $(0,05-0,36)$ & $(0,23-2,82)$ & $(0,11-0,24)$ & - \\
$\mathrm{N}$ & $\mathrm{n}=75$ & $\mathrm{n}=5$ & & $\mathrm{n}=6$ & $\mathrm{n}=9$ & $\mathrm{n}=2$ & $\mathrm{n}=1$ \\
$\mathrm{~T}$ & & & & & & & \\
$\mathrm{R}$ & & & & & & & \\
$\mathrm{O}$ & & & & & & - & - \\
$\mathrm{S}$ & $0,01 \pm 0,02$ & $0,03 \pm 0,01$ & $0,04 \pm 0,01$ & $0,03 \pm 0,01$ & - & - \\
$\mathrm{U}$ & $(0,01-0,15)$ & $(0,01-0,16)$ & $(0,01-0,13)$ & $(0,02-0,08)$ & & - \\
$\mathrm{R}$ & $\mathrm{n}=17$ & $\mathrm{n}=16$ & $\mathrm{n}=12$ & $\mathrm{n}=5$ & & - \\
\hline $\mathrm{N}$ & $0,12 \pm 0,01$ & - & - & - & $0,57 \pm 0,11$ & - & - \\
$\mathrm{O}$ & $(0,01-0,30$ & - & - & - & - & & \\
$\mathrm{R}$ & $\mathrm{n}=6$ & & & & & & \\
$\mathrm{~T}$ & & & & & & & \\
$\mathrm{E}$ & & & & & & & \\
\hline
\end{tabular}

n: número de muestras positivas

Tabla 6. Concentración media $(\mu \mathrm{g} / \mathrm{L} \pm \mathrm{s})$ de herbicidas en muestras positivas procedentes de fuentes, pozos y sondeos de áreas agrícolas del sur de Navarra. Entre paréntesis figura el intervalo de concentraciones de las muestras con residuos.

\begin{tabular}{cccccccc}
\hline Imazametabenz & Terbutilazina & Atrazina & Simazina & Isoproturon & Clortoluron & Terbutrina & Metribuzina \\
\hline $0,023 \pm 0,003$ & $0,06 \pm 0,02$ & $0,09 \pm 0,01$ & $0,04 \pm 0,01$ & $0,05 \pm 0,01$ & $0,24 \pm 0,01$ & $0,05 \pm 0,01$ & $0,06 \pm 0,01$ \\
$(0,01-0,05)$ & $(0,01-1,12)$ & $(0,01-0,40)$ & $(0,02-0,20)$ & $(0,05-0,06)$ & $(0,22-0,26)$ & $(0,01-0,19)$ & $(0,04-0,07)$ \\
$\mathrm{n}=9$ & $\mathrm{n}=39$ & $\mathrm{n}=60$ & $\mathrm{n}=36$ & $\mathrm{n}=2$ & $\mathrm{n}=3$ & $\mathrm{n}=5$ & $\mathrm{n}=2$ \\
\hline
\end{tabular}

n: número de muestras positivas

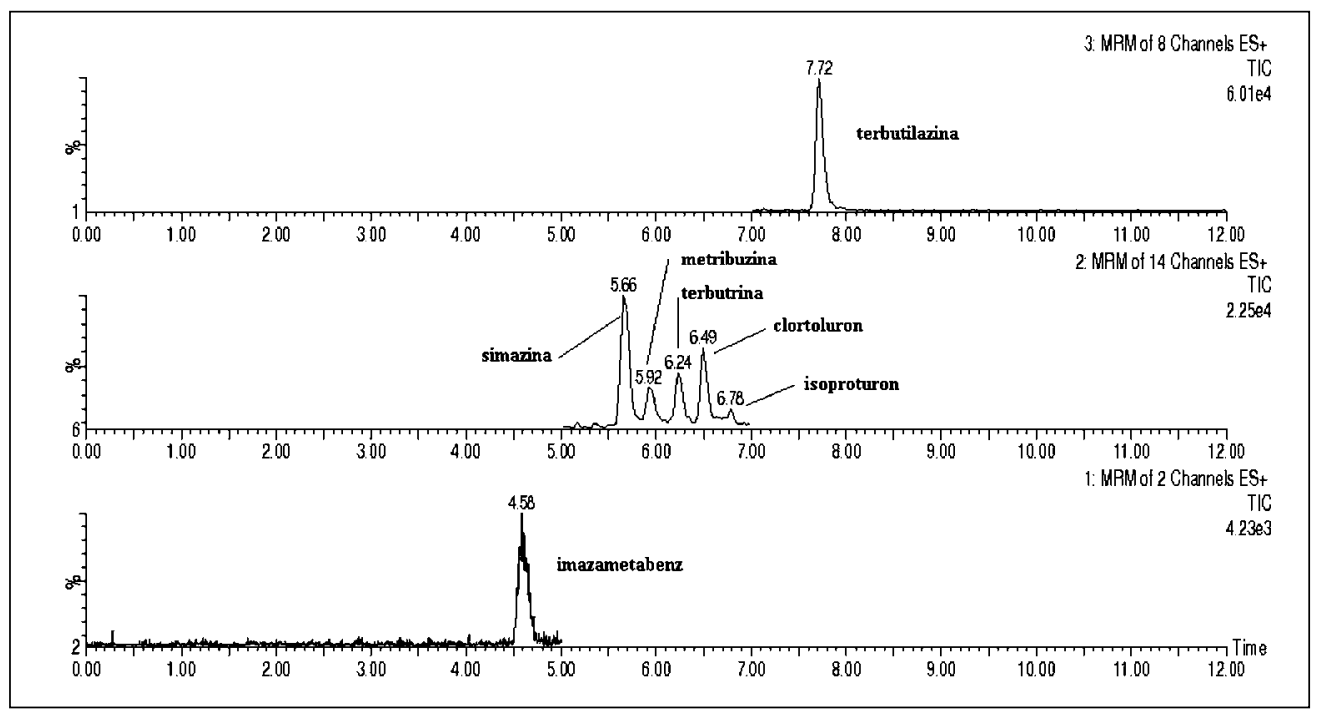

Figura 6. Cromatograma correspondiente a una muestra de agua de pozo de la zona sur. Las concentraciones halladas fueron: simazina $0,20 \pm 0,02 \mu \mathrm{g} / \mathrm{L}$; terbutilazina $0,13_{ \pm} 0,01 \mu \mathrm{g} / \mathrm{L}$; terbutrina $0,02 \pm 0,01 \mu \mathrm{g} / \mathrm{L}$; metribuzina $0,04 \pm 0,01 \mu \mathrm{g} / \mathrm{L}$; clortoluron $0,25 \pm 0,01 \mu \mathrm{g} / \mathrm{L}$; isoproturon $0,06 \pm 0,005 \mu \mathrm{g} / \mathrm{L}$; imazametabenz $0,02 \pm 0,002 \mu \mathrm{g} / \mathrm{L}$. 


\section{DISCUSIÓN}

La utilización de la técnica LC-MS/MS permitió ampliar el número de los compuestos analizados en un estudio previo realizado en Navarra ${ }^{9}$. Los límites de cuantificación obtenidos mediante LC-MS/MS para 11 de los 12 compuestos fueron entre 2 y 10 veces inferiores al límite legal. En el caso de pendimetalina, el límite de cuantificación coincidió con el límite legal $(0,1$ $\mu \mathrm{g} / \mathrm{L}$ ). El método analítico descrito resultó, por tanto, adecuado para el análisis de los herbicidas objeto de estudio, aunque debe citarse la excepción de glifosato. La determinación de este compuesto requiere una derivatización previa ${ }^{13}$ a su análisis mediante LC-MS/MS, lo que impidió su inclusión en el método multirresiduo propuesto.

Los resultados globales de Navarra, tanto en lo que respecta a los porcentajes de muestras de abastecimientos con residuos de herbicidas (33\%), como al de muestras que superaron el valor normativo (8\%), son similares a los encontrados por Schipper $\mathrm{y} \mathrm{col}^{14}$ en un estudio realizado sobre 771 muestras de muestras de agua subterránea de Holanda. Estos autores detectaron pesticidas en el $27 \%$ de las muestras y en el $11 \%$ de ellas se superó el límite normativo de $0,1 \mu \mathrm{g} / \mathrm{l}$. Otros autores ${ }^{15}$, en muestras de agua potable tomadas en zonas agrícolas de Polonia, encontraron residuos de atrazina en el $71 \%$ de las muestras (primavera de 2002) y de simazina en el $29 \%$ de las aguas (primavera de 2003). Estos porcentajes son superiores a los hallados en nuestro trabajo, pese a que los límites de detección proporcionados por la técnica utilizada por estos últimos investigadores (GC-ECD) son más altos (de 1 a $2 \mu \mathrm{g} / \mathrm{L}$ ) que los que ofrece la técnica LC-MS/MS empleada en nuestro estudio (de 0,01 a $0,1 \mu \mathrm{g} / \mathrm{L}$ ). De estos datos se desprende la conclusión de que, incluso en la zona centro de Navarra, -en la que el porcentaje de aguas de abastecimiento que superó los límites fue mayor (un 11\%)-, éste no fue más alto que los porcentajes de muestras no conformes encontrados en otros trabajos. En estudios recientes realizados sobre muestras de aguas superficiales procedentes del este de España, otros autores han encontrado residuos de pesticidas en un alto porcentaje de las muestras. Así, Planas y col informaron de que en el período estival, hallaron residuos en más del $50 \%$ de las muestras de aguas superficiales analizadas. En este trabajo, cuatro pesticidas (atrazina, terbutilazina, dicloroanilina y fenitrotion) superaron la concentración de $1 \mu \mathrm{g} / \mathrm{L}$. Otros autore ${ }^{16}$ detectaron herbicidas e insecticidas en el $78 \%$ de las muestras de agua de la desembocadura del río Ebro en Tarragona (España). Este porcentaje de muestras positivas es algo inferior al $86 \%$ encontrado en las muestras de agua de pozos y fuentes de zonas agrícolas del sur de Navarra de nuestro trabajo.

Las diferencias entre los resultados obtenidos para las tres zonas de Navarra pueden explicarse atendiendo a las diferencias climáticas, y por tanto de cultivos, que afectan a las prácticas agrícolas de cada región. El uso de herbicidas en la zona norte se produce en áreas muy limitadas, donde hay cultivos de cereal. A la inversa, esto se traduce en que en los abastecimientos del norte sin cultivos de cereal próximos, sea improbable la presencia de residuos de herbicidas. En la zona centro, con agricultura cerealista extensiva, los porcentajes de muestras positivas se incrementan notablemente. Los abastecimientos de esta zona en los que se detectan residuos (41\%) corresponden, típicamente, a pequeños núcleos rurales con cultivos de trigo o cebada. Parece, por tanto, que la aparición de residuos en las aguas de estas poblaciones está influida por las prácticas agrícolas, pero también por la vulnerabilidad de los abastecimientos. En la zona sur de Navarra se da, en las zonas de regadío, la agricultura intensiva. Los abastecimientos de esta zona que recurran a las aguas subterráneas podrían verse afectados por los residuos de herbicidas. En esta zona, el porcentaje de muestras en las que se detectan residuos (46\%) es similar al de la zona centro. No obstante, el porcentaje de muestras con residuos por encima del límite es menor que el encontrado en el centro de Navarra (7\% frente al $11 \%$ ). 
La concentración media de las muestras positivas de la de la zona sur (Tabla 5) fue la más baja de las tres zonas consideradas. También se aprecian diferencias entre los herbicidas encontrados en las zonas del estudio. En las demarcaciones norte y centro son mayoritarios los herbicidas asociados a los cultivos de cereal (imazametabenz, clortuoluron, isoproturon...) mientras que en el sur, los compuestos mayoritarios son los empleados en productos hortícolas y en el cultivo del maíz (terbutilazina, atrazina, simazina...). Estos resultados coinciden con los de Montoro y $\mathrm{col}^{17}$, que detectaron la presencia de triazinas en aguas procedentes de zonas de agricultura intensiva de Almería (España). No obstante, en la zona sur de Navarra, en la que los cultivos de cereal no son predominantes, también se detectó imazametabenz, aunque a concentraciones medias muy inferiores a las de las otras demarcaciones. En un estudio previo ${ }^{18}$ realizado en Montana (EEUU), se estableció que las concentraciones de imazametabenz en las aguas subterráneas están controladas por las recargas hidraúlicas de los métodos de riego, por su persistencia en el suelo, por las características hidráulicas del acuífero, y por la adsorción/desorción de imazametabenz en las partículas del suelo. Debe considerarse que este compuesto, principio activo del preparado comercial Assert, estuvo autorizado en España para su «uso esencial» hasta el mes de junio de 2007; su utilización quedó prohibida a partir del 31 de diciembre del mismo año. En Navarra, se utilizó atrazina en algún "uso esencial» hasta el año 2007, en el que dejó de ser materia activa autorizada.

Las notables diferencias entre las concentraciones de los herbicidas utilizados en los cultivos de cereal (zonas centro y norte) y las de los herbicidas de cultivos hortícolas (zona sur) pueden deberse a varias causas. Por una parte, la extensión de los cultivos de cereal es mayor que el de los productos hortícolas; por otra parte, las dosis recomendadas (entre 2 y $5 \mathrm{~kg} / \mathrm{Ha}$ ) de herbicidas como imazametabenz, clortoluron o isoproturon eran mayores que las de atrazina, simazina o terbutilazina (entre
1 y $2 \mathrm{~kg} / \mathrm{Ha})^{19}$. También puede pensarse que las diferencias en la insolación, el régimen de lluvias, y la geología de las diferentes áreas consideradas influyen en la contaminación por herbicidas de las aguas. Las diferencias en los herbicidas detectados $\mathrm{y}$ en sus concentraciones son mayores en un estudio realizado por Kuster y $\mathrm{col}^{16}$ en aguas superficiales del delta del río Ebro (Tarragona). Estos autores hallaron concentraciones altas de algunos herbicidas no detectados en nuestro estudio, como alacloro. Las concentraciones medias de atrazina $(0,314 \mu \mathrm{g} / \mathrm{L})$ fueron más elevadas que las encontradas en los abastecimientos y las zonas agrícolas de nuestro estudio $(0,04$ y $0,09 \mu \mathrm{g} / \mathrm{L}$, respectivamente), que se presentan en la tabla 6 . Por el contrario, las concentraciones medias de clortoluron $(0,003)$ e isoproturon $(0,008 \mu \mathrm{g} / \mathrm{L})$ fueron inferiores a las halladas en nuestro trabajo $(0,24 \mu \mathrm{g} / \mathrm{L}$ y $1,09 \mu \mathrm{g} / \mathrm{L})$. En aguas subterráneas procedentes de la región de Valencia (España), con predominio del cultivo de cítricos, Hernández y col $^{20}$ detectaron con mayor frecuencia simazina, terbutilazina, bromacilo, terbumeton, y diuron. Existen, por tanto, algunas diferencias cuali y cuantitativas entre los resultados de los estudios de Tarragona y Valencia y los obtenidos en nuestro trabajo. Estas diferencias pueden atribuirse a las diferencias en los cultivos predominantes en Navarra y en las áreas mediterráneas consideradas, y al distinto origen de las muestras de agua de los estudios.

La evolución temporal que se observó en los abastecimientos seleccionados de cada una de las zonas (Figs. 3 y 4 ) puede explicarse por las diferencias climáticas e hidrológicas de las áreas consideradas. Los abastecimientos de la zona norte y centro toman sus aguas de manantiales que drenan pequeños acuíferos formados por materiales sueltos (derrubios de ladera, suelos más o menos profundos, etc.) que se apoyan sobre materiales impermeables. El agua de lluvia que se infiltra circula por dichos terrenos, reapareciendo en superficie, con un recorrido relativamente corto y con escaso tiempo de tránsito. Ello se traduce, en general, en que las variaciones tempo- 
rales en la composición de este tipo de aguas (incluyendo los contaminantes) son más rápidas que las que se producen en las aguas subterráneas de acuíferos de mayor entidad, como los que se desarrollan en el sur, en los que existe un efecto regulador que amortigua las variaciones temporales en la hidrogeoquímica del agua. En las muestras procedentes de pozos y fuentes de zonas agrícolas del sur de Navarra, se encontraron los mismos herbicidas detectados en abastecimientos, aunque con mayor frecuencia y a concentraciones medias más elevadas. La persistencia (vida media) de atrazina y simazina en el tipo de suelos predominante en la zona es alta. Aunque la época de aplicación de atrazina es la primavera, se ha detectado en gran parte de los muestreos realizados a lo largo de todas las estaciones de 2006. La zona más afectada correspondió a la zona del acuífero aluvial del Ebro, entre Tudela y Cortes, coincidiendo con la zona más afectada por la contaminación por nitratos de origen agrario y que ha sido declarada como vulnerable a dicha contaminación. En esta zona concurren los dos factores fundamentales que condicionan la contaminación de las aguas subterráneas: un intenso desarrollo agrícola con cultivos de regadío (maíz) y una alta vulnerabilidad hidrogeológica del acuífero a la contaminación.

\section{Agradecimientos}

La realización de este estudio ha sido posible gracias a la colaboración del Servicio de Seguridad Alimentaria y de Sanidad Ambiental del Instituto de Salud Pública, de la Sección de Recursos Hídricos del Departamento de Desarrollo Rural y Medio Ambiente y la de todo el personal del Laboratorio de Salud Pública en los aspectos prácticos necesarios.

\section{BIBLIOGRAFÍA}

1. Carabias R, Rodríguez E, Fernández ME, SÁnchez FJ. Evaluation of surface-and ground- water pollution due to herbicides in agricultural areas of Zamora and Salamanca (Spain). J Chromatogr A 2000; 869: 471-480.
2. BEXFIELD LM. Decadal-scale changes of pesticides in ground water of the United States, 1993-2003. J Environ Qual 2008; 37: 226-239.

3. Gooddy DC, Bloomfield JP, Chilton PJ, Johnson AC, Williams RJ. Assessing herbicide concentrations in the saturated and unsaturated zone of a Chalk aquifer in southern England. Ground Water 2001, 39: 262-271.

4. Tappe W, Groeneweg J, Jantsch B. Diffuse atrazine pollution in german aquifers. Biodegradation 2002, 13: 3-10.

5. Conte E, Rossi E, Spera G, Pompi V, Carfi` F, SpaDONI AR et al. Presence of plant protection products in three agricultural areas of regione Lazio. Commun Agric Appl Biol Sci 2003, 68: 865-874.

6. Planas C, Puig A, Rivera J, Caixach J. Analysis of pesticides and metabolites in Spanish surface waters by isotope dilution gas chromatography/mass spectrometry with previous automated solid-phase extraction. Estimation of the uncertainty of the analytical results. J Chromatogr A 2006; 27: 242-252.

7. Morvan X, Mouvet C, Baran N, Gutierrez A. Pesticides in the groundwater of a spring draining a sandy aquifer: temporal variability of concentrations and fluxes. J Contam Hydrol 2006, 87: 176-190.

8. ViJver MG, Van`t Zelfoe M, Tamis WL, Musters KJ, DE SNoo GR. Spatial and temporal analysis of pesticides concentrations in surface water: pesticides atlas. J Environ Sci Health 2008; 43: 665-674.

9. Fraile P, Pérez de Ciriza JA, Sálz I, Izu M, GonzáLEZ E, RodRIgo JL. Residuos de contaminantes orgánicos de diferentes orígenes en Navarra. An Sist Sanit Navar 2005; 28: 247-256.

10. Hernández F, Sancho JV, Pozo O, Lara A, Pitarch E. Rapid direct determination of pesticides and metabolites in environmental water samples at sub-microg/l level by on-line solid-phase extraction-liquid chromatographyelectrospray tandem mass spectrometry. J Chromatogr A 2001; 939: 1-11.

11. SAncho JV, Pozo O, Hernández J. Liquid chromatography and tandem mass spectrometry: a powerful approach for the sensitive and rapid multiclass determination of pesticides and transformation products in water. Analyst 2004; 129: 38-44.

12. Kuster M, López de Alda M, Barceló D. Analysis of pesticides in water by liquid chromatography-tandem mass spectrometric techniques. Mass Spectrom Rev 2006; 25: 900-916. 
13. IBÁÑez M, Pozo OJ, SANCho JV, LóPEz FJ, HERNÁNDEZ F. Re-evaluation of glyphosate determination in water by liquid chromatography coupled to electrospray tandem mass spectrometry. J Chromatogr A 2006; 17: 51-55.

14. SCHIPPER PN, VisSERs MJ, vAN DER LindEN AM. Pesticides in groundwater and drinking water wells: overview of the situation in the Netherlands. Water Sci Technol 2008; 57: 1277-1286.

15. Badach H, NazimeK T, KamińSKa IA. Pesticide content in drinking water samples collected from orchard areas in central Poland. Ann Agric Environ Med 2007; 14: 109-114.

16. Kuster M, López de Alda MJ, Barata C, Raldúa D, BARCELó D. Analysis of 17 polar to semi-polar pesticides in the Ebro river delta during the main growing season of rice by automated on-line solid-phase extraction-liquid chromatography-tandem mass spectrometry. Talanta 2008; 75: 390-401.
17. Montoro EP, González RR, Frenich AG, Torres ME, VIDAL JL. Fast determination of herbicides in waters by ultra-performance liquid chromatography/tandem mass spectrometry. Rapid Commun Mass Spectrom 2007; 21: 3585-3592.

18. MILLER KJ. Irrigation methods and transport of imazamethabenz-methyl to ground water: Greenfied Bench, Montana. JAWRA 2005; 41: 257-265.

19. De Liñán, C. Vademecum de productos fitosanitarios y nutricionales 2008. Agrotécnica ediciones, Madrid, España. 2008.

20. Hernández F, Marín JM, Pozo OJ, Sancho JV, LóPEz FJ, Morell I. Pesticide residues and transformation products in groundwater from a Spanish agricultural region on the Mediterranean Coast. Int J Environ Anal Chem 2008; 88: 409-424. 